\title{
Multiphoton Radiative Recombination of Electron Assisted by Laser Field
}

\author{
M. Yu. Kuchiev and V. N. Ostrovskyt \\ School of Physics, University of New South Wales Sydney 2052, Australia
}

\begin{abstract}
In the presence of an intensive laser field the radiative recombination of the continuum electron into an atomic bound state generally is accompanied by absorption or emission of several laser quanta. The spectrum of emitted photons represents an equidistant pattern with the spacing equal to the laser frequency. The distribution of intensities in this spectrum is studied employing the Keldysh-type approximation, i.e. neglecting interaction of the impact electron with the atomic core in the initial continuum state. Within the adiabatic approximation the scale of emitted photon frequencies is subdivided into classically allowed and classically forbidden domains. The highest intensities correspond to emission frequencies close to the edges of classically allowed domain. The total cross section of electron recombination summed over all emitted photon channels exhibits negligible dependence on the laser field intensity.
\end{abstract}

PACS numbers: 32.80.Wr, 34.80.Lx, 34.50.Rk 


\section{INTRODUCTION}

It is well known that the laser plasma emits photons with frequencies which are different from the frequency of the incident laser beam. For a number of applications an emission of high energy photons is the most interesting phenomenon. The known mechanisms which can be responsible for the high energy photo-production could be identified as the following three ones: the high harmonic generation, laser stimulated bremsstrahlung and laser assisted recombination. These processes differ by the initial and final states of the active electron. In the harmonic generation the initial state electron occupies a (laser-dressed) atomic bound state, usually it is the ground atomic state. In the final state of this reaction the electron can occupy either the same bound state, or some excited or even ionized state. The laser stimulated bremsstrahlung is a free-free transition during which the electron is scattered by an atom in the laser field. During the scattering the electron emits a high-energy quantum. In the laser assisted recombination (LAR) the electron starts in the laser-dressed continuum, but ends up in the bound state. The process of harmonic generation is currently studied very actively with important advancements both in theory and experiment (for reviews see Refs. [1,2]). The laser stimulated bremsstrahlung plays the very important role in plasma physics, see recent experimental [3, ,4] and theoretical [5,6] works.

The subject of the present study is the LAR process. As far as we know, it has not yet received a proper attention in the literature, although its importance for kinetics of laser plasma and its emission spectrum was indicated before [7]. From the point of view of the high-energy photo-production the LAR possesses an advantage over the stimulated bremsstrahlung because in LAR the electron impact energy is totally transferred to the high energy quanta.

The conventional (laser-field free) radiative recombination of the continuum electron to the bound state is a well studied process which is inverse to the photoionization. The frequency of the emitted photon is uniquely defined by the energy conservation law. When a similar process occurs in the presence of an intensive laser field the radiation spectrum becomes much more richer since the recombination may be accompanied by absorption or emission of laser quanta. Therefore the emitted photon spectrum represents a sequence of equidistant lines separated by the laser frequency $\omega$. The recent review by Hahn [B] on the electron recombination mentions only one, very special version of LAR process, namely one-photon LAR when the laser is tuned in resonance with the energy of free-bound electron transition, and only emission of photons with this particular energy is considered. The study of this special case was initiated quite long ago [9 13] and remains active in connection with the processes in the storage rings [14 20], formation of positronium [9] and antihydrogen 2123 and even with possible cosmological manifestations [24. In all these theoretical studies the laser field was presumed to be weak and its influence on an initial and/or final electron states was neglected, except Refs. [7,23] which are commented below.

For production of high-energy photons it is very interesting to extend the mentioned above studies allowing for the multiphoton absorption during LAR. Obviously the multiphoton processes can happen with high probability only in a strong laser field. From this point of view there arises a necessary to fulfill a systematic study of LAR in a strong laser field in multiphoton regime. This paper makes a first step in this direction.

An additional, and rather unexpected inspiration for the present study arises from the 
fact that LAR comprises one of the steps in the three-step quantum scheme of high harmonic generation. This scheme has recently been firmly established, see Ref. [25] and bibliography therein. The major statement of [25] is that the high harmonic generation can be described as the multiphoton ionization of an atomic electron which is followed by the LAR of this electron with the parent atomic particle. From this point of view the LAR plays a role of 'a part' of the problem of the high harmonic generation, which is important not only for the dense laser plasma, but also for photo-production from individual atoms in strong laser fields, where the harmonic generation is the major source for high energy photons.

The present study is devoted mostly to the patterns of intensities in the emitted photon spectrum depending on the laser field strength. We comment also on the influence of laser field on the total recombination cross section. Here, as well as in other applications, the laser field is intensive and LAR proceeds in substantially multiphoton regime.

Consider an electron in the laser-dressed continuum state $\Phi_{\mathbf{p}}(t)$ with the translational momentum p. Its recombination to the bound state generally results in the emission of photons with the frequencies $\tilde{\Omega}_{M}$ defined from

$$
\tilde{\Omega}_{M}=\frac{1}{2} \mathbf{p}^{2}+\frac{F^{2}}{4 \omega^{2}}-\varepsilon_{a}+M \omega,
$$

where $\varepsilon_{a}$ is the quasienergy of the field-dressed bound state $\Phi_{a}(t), \mathbf{F}$ is the amplitude of the electric field strength in the laser wave, $F^{2} /\left(4 \omega^{2}\right)$ is the electron quiver energy in the laser field, $M$ is an integer. Hereafter we use atomic system of units unless stated otherwise. In the zero-laser-field limit $(F \rightarrow 0)$ only emission of the photon with the frequency

$$
\Omega_{F \rightarrow 0}=\frac{1}{2} \mathbf{p}^{2}+\left|E_{a}\right|
$$

is allowed with $E_{a}$ being the bound state energy. The presence of an intensive laser field makes possible multiphoton processes when laser quanta are absorbed from the field or transmitted to it, with the amplitude

$$
C_{M}(\mathbf{p})=\frac{1}{T} \int_{0}^{T} d t\left\langle\Phi_{a}(t)\left|\exp \left(i \tilde{\Omega}_{M} t\right) \hat{d}_{\boldsymbol{\epsilon}}\right| \Phi_{\mathbf{p}}(t)\right\rangle, \quad \hat{d}_{\boldsymbol{\epsilon}}=\boldsymbol{\epsilon} \cdot \mathbf{r}
$$

where $T=2 \pi / \omega$ is the laser field period, and in the dipole momentum operator $\hat{d}_{\boldsymbol{\epsilon}}$ the unit vector $\boldsymbol{\epsilon}$ selects polarization of emitted radiation. The LAR cross section is

$$
\sigma_{M}(\mathbf{p})=\frac{4}{3 p} \frac{\left(\tilde{\Omega}_{M}\right)^{3}}{c^{3}}\left|C_{M}(\mathbf{p})\right|^{2},
$$

where $c$ is the velocity of light. The cross section (1.4) refers to the process of spontaneous LAR, since it is presumed that incident electromagnetic field with the frequency $\tilde{\Omega}_{M}$ is absent. In case if such a probe field is present, generally it would be amplified in course of propagation through the medium containing free electrons. There is a number of theoretical works devoted to calculation of related gain in case of one-photon LAR. A recent paper by Zaretskii and Nersesov [7] explores the amplification in case of multiphoton LAR. Generally these studies imply some assumptions regarding the medium properties and result in the 
expressions for the rate of stimulated transitions via that of spontaneous transitions and some characteristics of laser beam and the experimental arrangement [10, 12,7]. The present paper provides analysis of spontaneous LAR whereas issues of radiation amplification are beyond its scope.

\section{KELDYSH-TYPE APPROXIMATION}

We develop the Keldysh-type approximation where the interaction of the continuum electron with the atomic core is neglected, i.e. the laser-dressed electron continuum state $\Phi_{\mathbf{p}}$ is approximated by the well-known Volkov state. The laser wave is assumed to be linear polarized with the electric field strength $\mathbf{F}(t)=\mathbf{F} \cos \omega t$. Explicit expression for the Volkov functions is conveniently cast as

$$
\begin{aligned}
& \Phi_{\mathbf{p}}(\mathbf{r}, t)=\chi_{\mathbf{p}}(\mathbf{r}, t) \exp \left(-i \bar{E}_{\mathbf{p}} t\right), \\
& \chi_{\mathbf{p}}(\mathbf{r}, t)=\exp \left\{i\left[\left(\mathbf{p}+\mathbf{k}_{t}\right) \mathbf{r}-\int_{0}^{t}\left(E_{\mathbf{p}}(\tau)-\bar{E}_{\mathbf{p}}\right) d \tau+\frac{\mathbf{p F}}{\omega^{2}}\right]\right\},
\end{aligned}
$$

where the factor $\chi_{\mathbf{p}}(\mathbf{r}, t)$ is time-periodic with the period $T$,

$$
\begin{aligned}
\mathbf{k}_{t} & =\frac{\mathbf{F}}{\omega} \sin \omega t \\
E_{\mathbf{p}}(t) & =\frac{1}{2}\left(\mathbf{p}+\mathbf{k}_{t}\right)^{2} \\
\bar{E}_{\mathbf{p}} & =\frac{1}{T} \int_{0}^{T} E_{\mathbf{p}}(\tau) d \tau=\frac{1}{2} p^{2}+\frac{F^{2}}{4 \omega^{2}} .
\end{aligned}
$$

For the final bound state the field-free expression is employed

$$
\begin{aligned}
\Phi_{a}(\mathbf{r}, t) & =\varphi_{a}(\mathbf{r}) \exp \left(-i E_{a} t\right), \\
H_{a} \varphi_{a}(\mathbf{r}) & =E_{a} \varphi_{a}(\mathbf{r}),
\end{aligned}
$$

where $H_{a}$ is the effective atomic Hamiltonian in the single active electron approximation. The final bound state (2.6) is always available if electron collides with a positive ion. In case of collision with a neutral atom we assume existence of a stable negative ion. By substituting formulae (2.1)-(2.6) into (1.3) one can see that the integrand is a periodic function of time

provided the emitted photon frequency $\tilde{\Omega}_{M}$ satisfies (1.1) with integer $M$ and $\varepsilon_{a}$ substituted by $E_{a}$. The lowest possible frequency of the emitted photon is $\eta \omega$, where

$$
\eta=\frac{1}{\omega}\left(\frac{1}{2} p^{2}-E_{a}+\frac{F^{2}}{4 \omega^{2}}\right)-\operatorname{Ent}\left[\frac{1}{\omega}\left(\frac{1}{2} p^{2}-E_{a}+\frac{F^{2}}{4 \omega^{2}}\right)\right]
$$

with $\operatorname{Ent}(x)$ being an integer part of $x(0 \leq \eta<1)$. In the subsequent development we redefine labeling of emitted photon channels and instead of $\tilde{\Omega}_{M}$ (1.1) employ the notation

$$
\Omega_{m}=(m+\eta) \omega \quad(m \geq 0)
$$


The new label $m$ differs from the old one $M$ by an additive integer. We find the labeling by $m$ more convenient since it is rigidly related to the low-frequency edge of the emitted photon spectrum: $m=0$ corresponds to the lowest photon frequency $\eta \omega$.

By using the Fourier transformation formula (1.3) is rewritten as

$$
C_{m}(\mathbf{p})=-\frac{1}{T} \int_{0}^{T} d t \exp \{i[(m+\eta) \omega t-S(t)]\} \tilde{\varphi}_{a}^{(\epsilon)}\left(-\mathbf{p}-\mathbf{k}_{t}\right),
$$

where $S(t)$ is the classical action

$$
S(t)=\frac{1}{2} \int^{t} d \tau\left(\mathbf{p}+\mathbf{k}_{\tau}\right)^{2}-E_{a} t
$$

The function $\tilde{\varphi}_{a}^{(\epsilon)}(\mathbf{q})$ is defined as

$$
\tilde{\varphi}_{a}^{(\epsilon)}(\mathbf{q})=i\left(\boldsymbol{\epsilon} \cdot \nabla_{\mathbf{q}}\right) \tilde{\varphi}_{a}(\mathbf{q})
$$

where $\tilde{\varphi}_{a}(\mathbf{q})$ is the Fourier transform of the bound state wave function $\phi_{a}(\mathbf{r})$ :

$$
\tilde{\varphi}_{a}(\mathbf{q})=\int d^{3} \mathbf{r} \exp (-i \mathbf{q r}) \phi_{a}(\mathbf{r})
$$

For the bound state wave function we use an asymptotic expression

$$
\phi_{a}(\mathbf{r}) \approx A_{a} r^{\nu-1} \exp (-\kappa r) Y_{l m}(\hat{\mathbf{r}}) \quad(r \gg 1 / \kappa),
$$

where $\kappa=\sqrt{2\left|E_{a}\right|}, \nu=Z / \kappa, Z$ is the charge of the atomic residual core $(\nu=Z=0$ for a negative ion), $l$ is the active electron orbital momentum in the initial state and $\hat{\mathbf{r}}$ is the unit vector. The coefficients $A_{a}$ are tabulated for many negative ions [26]. The Fourier transform $\tilde{\varphi}_{a}(\mathbf{q})(2.13)$ is singular at $q^{2}=\kappa^{2}$ with the asymptotic behavior for $q \rightarrow \pm i \kappa$ defined by the long-range asymptote (2.14) in the coordinate space

$$
\tilde{\varphi}_{a}(\mathbf{q})=4 \pi A_{a}( \pm 1)^{l} Y_{l m}(\hat{\mathbf{q}}) \frac{(2 \kappa)^{\nu} \Gamma(\nu+1)}{\left(q^{2}+\kappa^{2}\right)^{\nu+1}}
$$

where $( \pm 1)^{l}$ corresponds to $q \rightarrow \pm i \kappa$. In particular, for a negative ion $(\nu=0)$ with the active electron in an $s$ state $(l=0)$ we have from (2.15)

$$
\begin{aligned}
\tilde{\varphi}_{a}(\mathbf{q}) & =\sqrt{4 \pi} A_{a} \frac{1}{\left(q^{2}+\kappa^{2}\right)} \\
\tilde{\varphi}_{a}^{(\epsilon)}(\mathbf{q}) & =-i(\boldsymbol{\epsilon} \cdot \hat{\mathbf{q}}) \sqrt{4 \pi} A_{a} \frac{2 q}{\left(q^{2}+\kappa^{2}\right)^{2}}
\end{aligned}
$$

$(\hat{\mathbf{q}} \equiv \mathbf{q} / q$ is unit vector $)$. 


\section{ADIABATIC APPROACH TO STIMULATED RECOMBINATION}

The time integral in (2.10) can be evaluated using the saddle point method. This amounts to the adiabatic approximation when the phase $(m+\eta) \omega t-S(t)$ in $(2.10)$ is assumed to be large. The position of saddle points in the complex $t$-plane is governed by equation

$$
S^{\prime}\left(t_{m \mu}\right)-\Omega_{m}=0
$$

or, more explicitly,

$$
\frac{1}{2}\left(\mathbf{p}+\mathbf{k}_{t_{m \mu}}\right)^{2}=E_{a}+(m+\eta) \omega
$$

It is convenient to single out in the electron momentum vector $\mathbf{p}=\mathbf{p}_{\|}+\mathbf{p}_{\perp}$ components parallel $\left(\mathbf{p}_{\|}\right)$and perpendicular $\left(\mathbf{p}_{\perp}\right)$ to the electric field vector $\mathbf{F}$. Then Eq. (3.2) is rewritten as

$$
\frac{1}{2}\left(p_{\|}+k_{t_{m \mu}}\right)^{2}=E_{a}-\frac{1}{2} p_{\perp}^{2}+(m+\eta) \omega
$$

For each value of $m$ this equation has a number of solutions $t_{m \mu}$ distinguished by the extra subscripts $\mu$. In the saddle point approximation the time integration in formula (1.3) is cast as

$$
C_{m}(\mathbf{p})=-\frac{1}{T} \sum_{\mu} \sqrt{\frac{2 \pi}{i S^{\prime \prime}\left(t_{m \mu}\right)}} \exp \left\{i\left[\Omega_{m} t_{m \mu}-S\left(t_{m \mu}\right)\right]\right\} \tilde{\varphi}_{a}^{(\epsilon)}\left(-\mathbf{p}-\mathbf{k}_{t_{m \mu}}\right)
$$

where summation is to be taken over the saddle points $t_{m \mu}$ operative in the contour integration $\left.\left[\mathbf{k}_{t_{m \mu}}=(\mathbf{F} / \omega) \sin \omega t_{m \mu}\right]\right)$.

The saddle points are found from Eq.(3.3) as

$$
\sin \omega t_{m \mu}=\frac{\omega}{F}\left(-p_{\|} \pm \sqrt{2(m+\eta) \omega-\kappa^{2}-p_{\perp}^{2}}\right) .
$$

The subscript $\mu$ labels solutions differing by the choice of the sign in (3.5) and sign in $\cos \omega t_{m \mu}= \pm \sqrt{1-\sin ^{2} \omega t_{m \mu}}$. There are four solutions per the laser field cycle (i.e for $\left.0 \leq \operatorname{Re} t_{m \mu}<T\right)$.

In order to elucidate the meaning of the saddle point equation (3.3) we rewrite it as

$$
E_{\mathrm{p}}\left(t_{m \mu}\right)-E_{a}=\Omega_{m}
$$

It shows that the photons are preferentially emitted at the moment of time when instantaneous continuum electron energy $E_{\mathrm{p}}(t)$ (2.4) is separated from the bound state energy $E_{a}$ by the energy of the emitted photon $(m+\eta) \omega$. The LAR process is most effective when this occurs at some real moment of time, i.e. the saddle points $t_{m \mu}$ are real-valued. This regime corresponds to the classically allowed radiation. It can happen only for some part of the emitted photon spectrum, i.e. only in some domain of $m$. Outside it, when $t_{m \mu}$ possesses an imaginary part, the emission is strongly suppressed. Remarkably, within the classically allowed domain the intensity of emitted lines could vary very significantly as detailed below. 
The necessary condition of classically allowed radiation,

$$
\Omega_{m}>\left|E_{a}\right|+\frac{1}{2} p_{\perp}^{2}
$$

makes real the right hand side of formula (3.5). Details of classically allowed emission depend on the relation between the electron translational momentum component $p_{\|}$and the momentum $F / \omega$ acquired by the electron in its quiver motion in the laser field. In the fast electron regime, $p_{\|}>F / \omega$, the term $\frac{1}{2}\left(p_{\|}+k_{t}\right)^{2}$ never passes zero as time $t$ varies. As a result, the saddle point equation (3.2) has two or zero real-valued solutions per field cycle (in the classically allowed and forbidden domains respectively, see Fig. 11a). In the slow electron case, $p_{\|}<F / \omega$, the $\frac{1}{2}\left(p_{\|}+k_{t}\right)^{2}$ passes via zero. Due to this circumstance, as seen from Fig. [1]b, for some interval of photon frequencies $\Omega_{m}$ the equation (3.2) has four real-valued solutions whereas for higher $\Omega_{m}$ only two solutions exist. Consequently, in this case the classically allowed domain is subdivided in two parts. The related LAR regimes are discussed below in more detail.

\section{A. Fast electron regime: $p_{\|}>F / \omega$}

Here one has to choose the upper sign in formula (3.5) in order to get a real-valued saddle point. The condition $\left|\sin \omega t_{m \mu}\right| \leq 1$ is straightforwardly reduced to

$$
\frac{1}{2}\left(p_{\|}-\frac{F}{\omega}\right)^{2}+\left|E_{a}\right|+\frac{1}{2} p_{\perp}^{2} \leq \Omega_{m} \leq \frac{1}{2}\left(p_{\|}+\frac{F}{\omega}\right)^{2}+\left|E_{a}\right|+\frac{1}{2} p_{\perp}^{2} .
$$

In this photon frequency interval only one pair of real saddle points $t_{m \mu}$ exists per field cycle, see Fig 1. These two saddle points are to be included into summation over $\mu$ in (3.4). The phase difference between the two terms in (3.4) varies with $m$. As a result $\left|C_{m}(\mathbf{p})\right|^{2}$ oscillates between zero and some envelope function $\Xi(m)$ defined as

$$
\begin{array}{r}
\Xi(m)=\frac{8 \pi}{T^{2} S^{\prime \prime}}\left|\tilde{\varphi}_{a}^{(\epsilon)}\left(-\mathbf{p}-\mathbf{k}_{t_{m \mu}}\right)\right|^{2}, \\
\left|\tilde{\varphi}_{a}^{(\epsilon)}\left(-\mathbf{p}-\mathbf{k}_{t_{m \mu}}\right)\right|^{2}=\pi A_{a}^{2} \frac{2(m+\nu) \omega-\kappa^{2}-p_{\perp}^{2}}{(m+\nu)^{4} \omega^{4}}, \\
S^{\prime \prime}=F \sqrt{2(m+\nu) \omega-\kappa^{2}-p_{\perp}^{2}} \sqrt{1-\frac{\omega^{2}}{F^{2}}\left(p_{\|}-\sqrt{2(m+\nu) \omega-\kappa^{2}-p_{\perp}^{2}}\right)^{2}} .
\end{array}
$$

As could be anticipated, the function $\Xi(m)$ has weak singularities at the boundaries of the classically allowed region. The extension of the classically allowed region on the photon frequency scale is $2 p_{\|} F / \omega$ with its center located at $\Omega_{\mathrm{c}}=\frac{1}{2} p^{2}+\left|E_{a}\right|+F^{2} /\left(2 \omega^{2}\right)$. For vanishing laser field $\Omega_{\mathrm{c}}$ tends to the limit (1.2) and the classically allowed domain shrinks to the single line. The condition that a single line dominates in the photon spectrum could be formulated as $2 p_{\|} F / \omega^{2} \sim 1$.

Fig. 2 illustrates evolution of the spectrum pattern with the laser intensity $I$. We consider electrons with the energy $E_{\mathrm{el}}=\frac{1}{2} p^{2}$ equal to $1 \mathrm{eV}(p=0.271)$ in the laser field with the frequency $\omega=0.0043$ and different intensities. The electron momentum $\mathbf{p}$ is directed along the laser field strength $\mathbf{F}\left(p_{\perp}=0\right)$. The electron recombines to the bound state of $\mathrm{H}^{-}$ion 
$\left(\kappa=0.2354, A_{a}=0.75[26]\right)$. The emission amplitudes are obtained by numerical evaluation of the time integral in (2.10). The laser field intensities $I=10^{11}, 10^{10}, 10^{9}, 10^{8}, 10^{7} \mathrm{~W} / \mathrm{cm}^{2}$ corresponds to the values of parameter $2 p F / \omega^{2}$ respectively $49.4,15.6,4.94,1.56,0.494$. For the weakest field considered $\left(I=10^{7} \mathrm{~W} / \mathrm{cm}^{2}\right)$ the intensity of the principal line in the spectrum $(m=14)$ exceeds more than 50 times these of adjacent satellites. For $I=10^{8}$ $\mathrm{W} / \mathrm{cm}^{2}$ this ratio is substantially smaller $(\sim 5)$. When laser field is increased by an order of magnitude, the dip in the emitted photon spectrum appears at $m=15$. This is the first manifestation of the oscillatory structure in the spectrum due to interference of two contributions in (3.4). For $I=10^{10} \mathrm{~W} / \mathrm{cm}^{2}$ the structure becomes well manifested. At last, for $I=10^{11} \mathrm{~W} / \mathrm{cm}^{2}$ the structure becomes well developed and extended. In the latter case, in fact, the situation is beyond the fast electron regime; it will be discussed in the next subsection.

The semiclassical formula (3.4) is applicable when the classically allowed domain is sufficiently broad on the frequency scale. Fig. 3 shows the photon spectrum in the well manifested semiclassical regime $\left(E_{\mathrm{el}}=10 \mathrm{eV}, I=10^{11} \mathrm{~W} / \mathrm{cm}^{2}, p=0.857, F / \omega=0.392\right)$. In the classically allowed domain $(31 \leq m \leq 187)$ the quantities $\left|C_{m}(p)\right|^{2}$ obtained by numerical evaluation of the integral (2.10) over time (circles) oscillate violently due to the interference effects. Outside this region $\left|C_{m}(p)\right|^{2}$ decrease very rapidly. Note that the most efficient emission occurs at the edges of the classically allowed interval. This effect is completely analogous to enhancement of the probability density near the turning points for the quantum particle moving in the potential well. The envelope function (3.9) (solid curve) reproduces well this overall behavior. The saddle point approximation (3.4) allows us to reproduce well the oscillatory structure (squares in Fig. 3). Within the classically-allowed domain the summation in this formula runs over two real-valued saddle points $t_{m \mu}$. As $m$ varies approaching the domain border, two saddle points lying at the real- $t$ axis approach each other and eventually merge at the boundary. After that they separate again moving perpendicular to the real axis in the complex $t$-plane. The latter situation corresponds to the classically forbidden, or tunneling regime where only one saddle point is to be included in the summation over $\mu$ in (3.5) (namely, that which ensures exponential decrease of $\left|C_{m}(p)\right|^{2}$ outside the classically-allowed domain). The transition between two regimes could be described by the Airy function. We do not pursue here the detailed description of this, rather standard situation. In particular, Fig. 3, the results shown by squares in Fig. 3 are obtained using the plain semiclassical formula (3.4) with two or one saddle points included as discussed above; the deviations from the numerical results are seen to be essential only in a very narrow transitions region. Since the numerical evaluation of integral (2.10) over time is not difficult, we employ the adiabatic approach in order to obtain better insight into the pattern of emitted radiation spectrum, but not for producing an alternative method to evaluate the amplitudes.

\section{B. Slow electron regime: $p_{\|}<F / \omega$}

In this case the real-valued result for $t_{m \mu}$ is provided by both upper and lower sign in the expression (3.5). It is easy to see from Fig. [1 b that the classically allowed region of photon frequencies is subdivided in two domains. The first of them, with one pair of realvalued saddle points $t_{m \mu}$, corresponds to $\Omega_{m}$ lying in the interval (3.8). At smaller photon 
frequencies, another subdomain is defined by the condition

$$
\left|E_{a}\right|+\frac{1}{2} p_{\perp}^{2} \leq \Omega_{m} \leq \frac{1}{2}\left(p_{\|}-\frac{F}{\omega}\right)^{2}+\left|E_{a}\right|+\frac{1}{2} p_{\perp}^{2} .
$$

Here two pairs of real saddle points $t_{m \mu}$ exist. The spectrum for this situation is illustrated by Fig. 田 $\left(E_{\mathrm{el}}=0.1 \mathrm{eV}, I=10^{11} \mathrm{~W} / \mathrm{cm}^{2}, p=0.0857, F / \omega=0.392\right)$. The classically allowed domain lies in the interval $7 \leq m \leq 32$, with the four-saddle-point regime being operative for $7 \leq m \leq 17$, and the two-saddle point regime for $18 \leq m \leq 32$. The results of numerical calculations shown by circles suggest that the oscillations in $\left|C_{m}(p)\right|^{2}$ or $\sigma_{m}(p)$ proceed with two different frequencies, the higher frequency being characteristic for the four-saddle-point domain. The plain semiclassical formula (3.4) (squares) essentially reproduces this structure. Of course, it is not designed for accurate description of a transition between the two-saddlepoint and four-saddle-point regimes where the deviations are seen to be larger. A special, more sophisticated treatment is required here, but such complications are not pursued in the present study as argued above. The non-standard situation emerges also at the left edge of the classically allowed interval where all saddle points simultaneously move from the real axis into the complex $t$ plane. This transition region could not be described by a simple Airy-type pattern that is known to give a monotonous decrease in the classically forbidden domain; on the contrary, the numerical results reveal some structure in this region, see Fig. 4. Bearing all this in mind it is not unexpected that the plain semiclassical approximation (3.4) essentially fails near the left border of the classically allowed domain.

It is worthwhile to mention also another region where the standard semiclassical approximation fails. Namely, for $\Omega=0$ the saddle point positions coincide with the poles of the function $\tilde{\varphi}_{a}^{(\epsilon)}$. The situation when an exact coincidence occurs is tractable rather easily [27]. Somewhat more effort is required to obtain uniform description of a transition between this case and a situation when the saddle point and the pole are well separated, as presumed in simple formula (3.4). Again, such sophistication are beyond the scope of the present study.

At last, Fig. 5 shows a transient situation between the fast and slow electron regimes $\left(E_{\mathrm{el}}=1 \mathrm{eV}, I=10^{11} \mathrm{~W} / \mathrm{cm}^{2}, p=0.271, F / \omega=0.392\right)$. Here only two harmonics $(m=7,8)$ correspond to the four-saddle-point regime. The remaining part of the classically-allowed domain, $9 \leq m \leq 56$ corresponds to two-saddle-point regime. Most of the spectrum is well described by the plain saddle-point approximation (3.4) and covered by the envelope function (3.9), albeit the highest peak at $m=9$ exceeds it, as being in the region of the transition between the two and four-saddle point regimes. Quite paradoxically, the lowfrequency classically forbidden region with well manifested structure exhibits much higher emission intensities as compared with the large-frequency edge of the classically allowed domain.

\section{CONCLUSION}

As discussed in the Introduction, the LAR is one of the processes responsible for emission of high energy photons by the laser plasma. Surprisingly, it has not yet received attention of researchers. This is particularly unsatisfactory since the other processes leading to high energy photons (harmonic generation and laser stimulated bremsstrahlung) are currently under active scrutiny. The present paper could be considered as a first step to start filling 
this gap. The theory in many aspects is parallel to the treatment of multiphoton ionization (MPI) where the Keldysh approximation is known to provide an important insight and quantitatively reliable results. The origin of differences between MPI and LAR lies in the kinematics: in MPI process the allowed electron energy in the continuum are robustly defined by the parameters of the system (initial electron binding energy, laser field frequency and strength), whereas in the LAR the continuum electron energy is arbitrary. This rather trivial observation results in important consequences of physical character. They are particularly lucid in the adiabatic regime when laser frequency is sufficiently small. The ionization is a tunneling process for all above-threshold channels. On the contrary, in the LAR there is a domain of photon frequencies for which emission is allowed classically.

The Keldysh-type approximation allowed us to describe evolution of the LAR spectrum as the laser field varies, from the single line with only weak satellites in the low-field limit to the broad pattern of equidistantly spaced harmonics in the strong field case. In the adiabatic approximation (i.e. the saddle point method) the photon spectrum is subdivided into classically allowed and classically forbidden domains, with the line intensities being highest at the boundaries of the former region. Concerning the quantitative side of the problem, the adiabatic approach is less efficient for the LAR process as compared with the treatment of above threshold ionization (ATI). The reason is that in the latter case the saddle point method is well applicable in its most simple form, whereas for LAR process some technical complications emerge. The difference stems from the fact that ATI process always effectively occurs at complex-valued moments of time, whereas for LAR this is generally not the case, and several regimes could be operative with the transition regions between them. Albeit not drastic, these complications to our opinion hardly warrant necessary cumbersome analytical involvements, bearing in mind that the numerical calculations are quite simple and straightforward. Nevertheless the saddle point method remains very useful for understanding the intensity patterns in the emitted photon spectrum.

An additional assumption of the present study, that in principle could be easily abandoned, is the use of asymptotic expression (2.14) for the final bound state wave function. Again, in the LAR process the situation is less favorable for this approximation as compared with the ATI process. This is because, as discussed in detail earlier [27], the long-range asymptote of the bound state wave function governs ATI amplitudes, whereas LAR process is more sensitive to the wave function behavior in the entire coordinate space.

As is pictured by Fig. 2, the amount of noticeable lines in the photon spectrum increases with the laser field strength, but the intensity of each individual line decreases in average. The cross section of the electron transition into the bound state summed over all emitted photon channels is $\sigma_{\text {tot }}(p)=\sum_{m>0} \sigma_{m}(p)$. It exhibits only very weak dependence on the laser filed intensity $I$ 28]. For instance, in the particular case of Fig. 2 we obtain for $\sigma_{\text {tot }}(p)$ the values $3.85 \cdot 10^{-6}, 3.85 \cdot 10^{-6}, 3.89 \cdot 10^{-6}, 3.64 \cdot 10^{-6}, 3.4 \cdot 10^{-6}$ for the laser field intensities $I=10^{11}, 10^{10}, 10^{9}, 10^{8}, 10^{7} \mathrm{~W} / \mathrm{cm}^{2}$. Recent calculations 23 of the laser-assisted antihydrogen formation in positron-antiproton collisions employed Coulomb-Volkov wave function for the initial electron continuum state $\Phi_{\mathbf{p}}$ and the laser-perturbed wave function for the bound state. The authors considered only one-photon LAR process and concluded that the LAR cross section decreases for the stronger laser fields. The present results indicate that if the multiphoton processes are included, then the total LAR cross section is essentially independent on laser field intensity. 
Thus the effect of a laser on the recombination process looks very straightforward. The total cross section of recombination essentially is not changed by a laser field, but is redistributed over equidistant pattern in photon spectrum that becomes broader as the laser intensity increases.

\section{ACKNOWLEDGMENTS}

This work has been supported by the Australian Research Council. V. N. O. acknowledges the hospitality of the staff of the School of Physics of UNSW where this work has been carried out. 


\section{REFERENCES}

* Permanent address: Institute of Physics, The University of St Petersburg, 198904 St Petersburg, Russia; E-mail: Valentin.Ostrovsky@pobox.spbu.ru

[1] M. Protopapas, C. H. Keitel, and P. L. Knight, Rep. Progr. Phys. 60, 389 (1997).

[2] V. T. Platonenko and V. V. Strelkov, Kvantovaya Elektronika 25, 582 (1998) [Quantum Electronics 28, 584 (1998)].

[3] Y. Ueshima, Y. Kishimoto, A. Sasaki, T. Tajima, Laser Part. Beams. 17, 45 (1999).

[4] P. A. Norreys M. Santala, E. Clark, M. Zepf, I. Watts. F. N. Beg, K. Krushelnick, M. Tatarakis, A. E. Dangor, X. Fang, P. Graham, T. McCanny, R. P. Singhal, K. W. D. Ledingham, A. Creswell, D. C. W. Sanderson, J. Magill, A. Machacek, J. S. Wark, R. Allott, B. Kennedy, D. Neely. Phys. Plasmas. 62150 (1999).

[5] V. P. Silin, Izv. Akad. Nauk Ser. Fiz. 63, 707 (1999).

[6] V. A. Astapenko, Laser Phys. 8, 1066 (1998).

[7] D. F. Zaretskii and E. A. Nersesov, Zh. Eksp. Teor. Fiz. 109, 1994 (1996) [JETP 82, $1073(1996)]$.

[8] Y. Hahn, Rep. Progr. Phys. 60, 691 (1997).

[9] L. A. Rivlin, Kvantovaya Elektronika 6, 594 (1979) [Sov. J. Quant. Electron 9, 353 (1979)].

[10] F. H. M. Faisal, A. Lami, and N.K.Rahman, J. Phys. B 14, L569 (1981); A. Lami, N.K.Rahman, and F. H. M. Faisal, Phys. Rev. A 30, 2433 (1984).

[11] R. Neumann, H. Poth, A. Winnacker, and A. Wolf, Z. Phys. A 313, 253 (1983).

[12] B. Ritchie, Phys. Rev. A 30, 1849 (1984).

[13] E. F. Fill, Phys. Rev. Lett. 56, 1687 (1986).

[14] U. Schramm, J. Berger, M. Grieser, D. Habs, E. Jaeschke, G. Kilgus, D. Schwalm, and A. Wolf, Phys. Rev. Lett. 67, 22 (1991).

[15] F. B. Yousif, P. Van der Donk, Z. Kucherovsky, J. Reiss, E. Brannen, J. B. A. Mitchell, and T. J. Morgan, Phys. Rev. Lett. 67, 26 (1991).

[16] U. Schramm, T. Schlüssler, D. Habs, D. Schwalm, and A. Wolf, Hyperfine Interactions 99, 309 (1996).

[17] S. Pastuszka, U. Schramm, M. Grieser, C. Broude, R. Grimm, D. Habs, J. Kenntner, H.-J. Miesner, T. Schüssler, D. Schwalm, and A. Wolf, Nucl. Inst. Meth. A 369, 11 (1996).

[18] S. Asp, R. Schuch, D. R. DeWitt, C. Biedermann, H. Gao, W. Zong, G. Andler, E. Justiniano, Nucl. Inst. Meth. B 117, 31 (1996).

[19] M. L. Rogelstad, F. B. Yousif, T. J. Morgan, and J. B. A. Mitchell, J. Phys. B 30, 3913 (1997)

[20] E. Justiniano, G.Andler, S. Asp, D. R. DeWitt, and R.Schuch, Hyperfine Interactions 108, 283 (1997).

[21] R. Neumann, H. Poth, A. Winnacker, and A. Wolf, Z. Phys. A 313, 253 (1983).

[22] A. Müller and A. Wolf, Hyperfine Interactions 109, 233 (1997).

[23] S.-M. Li, Y.-G. Miao, Z.-F. Zhou, J. Chen and Y.-Y. Liu, Phys. Rev. A 58, 2615 (1998).

[24] W. Klemperer, X.-C. Luo, R. Rosner, and D. N. Schramm, Proc. Nat Ac. Sci. USA 92, 6166 (1995).

[25] M. Yu. Kuchiev and V. N. Ostrovsky, J.Phys.B 32, L189 (1999); Phys. Rev. A (accepted for publication). 
[26] A. A. Radzig and B. M. Smirnov Reference Data on Atoms, Molecules and Ions (Berlin: Springer, 1985). Unfortunately the numerical value of the asymptotic parameter $A$ for $\mathrm{H}^{-}$ion is absent in the standard reference book [A. A. Radzig and B. M. Smirnov, Reference Data on Atoms, Molecules and Ions (Berlin: Springer, 1985)]. In our calculations, as previously [27] [25], we assume $A=0.75$ as given by V. M. Galitzkii, E. E. Nikitin, and B. M. Smirnov, Teoriya Stolknovenii Atomnykh Chastitz (In Russian: Theory of Atomic Particle Collisions) (Moscow: Nauka, 1981).

[27] G. F. Gribakin and M. Yu. Kuchiev, Phys. Rev. A 55, 3760 (1997); J. Phys. B 30, L657 (1997); 31, 3087 (1998); M. Yu. Kuchiev and V. N. Ostrovsky, J. Phys. B 31, 2525 (1998).

[28] Unfortunately currently we did not succeed in analytical derivation of this result. 


\section{FIGURES}

FIG. 1. Regimes of fast $\left(p_{\|}>F / \omega\right)$ and slow $\left(p_{\|}<F / \omega\right)$ electron in the laser-assisted recombination process. For each regime the schematic plots show electron momentum with account for the quiver motion in laser field $\Pi(t)_{\|} \equiv p_{\|}+(F / \omega) \sin \omega t$ and the effective instantaneous kinetic energy $\frac{1}{2} \Pi(t)_{\|}$. As time $t$ varies, the function $\frac{1}{2} \Pi(t)_{\|}$oscillates in the interval that covers the emitted photon energies $\Omega_{m}$ allowed for population classically. Outside this interval only non-classical (tunneling) population is possible. Fig. 17a shows that in the classically allowed domain each value of the photon energy $\Omega$ is passed twice during the laser field period $T$ if the electron is fast $\left(p_{\|}>F / \omega\right)$. In the slow electron regime $\left(p_{\|}<F / \omega\right)$ the classically allowed domain of $\Omega_{m}$ is subdivided into two regions, as seen from Fig. 1. The photons with higher $\Omega_{m}$ are again emitted in the double-passage mode, whereas the lower values of $\Omega_{m}$ are passed four times per the laser field cycle.

FIG. 2. Factor $\left|C_{m}(p)\right|^{2}$ and cross section $\sigma_{m}(p)$ for laser-assisted recombination of the electron with the energy $E_{\mathrm{el}}=1 \mathrm{eV}$ to the bound state in $\mathrm{H}^{-}$ion. The results of numerical integration in Eq.(2.10) are shown for the laser field with the frequency $\omega=0.0043$ and the intensities $I=10^{7}$ $\mathrm{W} / \mathrm{cm}^{2}$ (crosses); $10^{8} \mathrm{~W} / \mathrm{cm}^{2}$ (triangles); $10^{9} \mathrm{~W} / \mathrm{cm}^{2}$ (diamonds); $10^{10} \mathrm{~W} / \mathrm{cm}^{2}$ (squares) and $10^{11}$ $\mathrm{W} / \mathrm{cm}^{2}$ (circles). The symbols are joined by lines to help the eye.

FIG. 3. Same as in Fig. 2, but for the electron energy $E_{\mathrm{el}}=10 \mathrm{eV}$ and the laser field intensity $I=10^{11} \mathrm{~W} / \mathrm{cm}^{2}$. The results of numerical calculations and plain semiclassical formula (3.4) are shown respectively by circles and squares. The semiclassical envelope function (3.9) is given by solid line. In the zero-laser-field limit the spectrum shrinks to the single line with the position indicated by vertical arrow.

FIG. 4. Same as in Fig. 3, but for the electron energy $E_{\mathrm{el}}=0.1 \mathrm{eV}$ and the laser field intensity $I=10^{11} \mathrm{~W} / \mathrm{cm}^{2}$.

FIG. 5. Same as in Fig. 3, but for the electron energy $E_{\mathrm{el}}=1 \mathrm{eV}$ and the laser field intensity $I=10^{11} \mathrm{~W} / \mathrm{cm}^{2}$. 

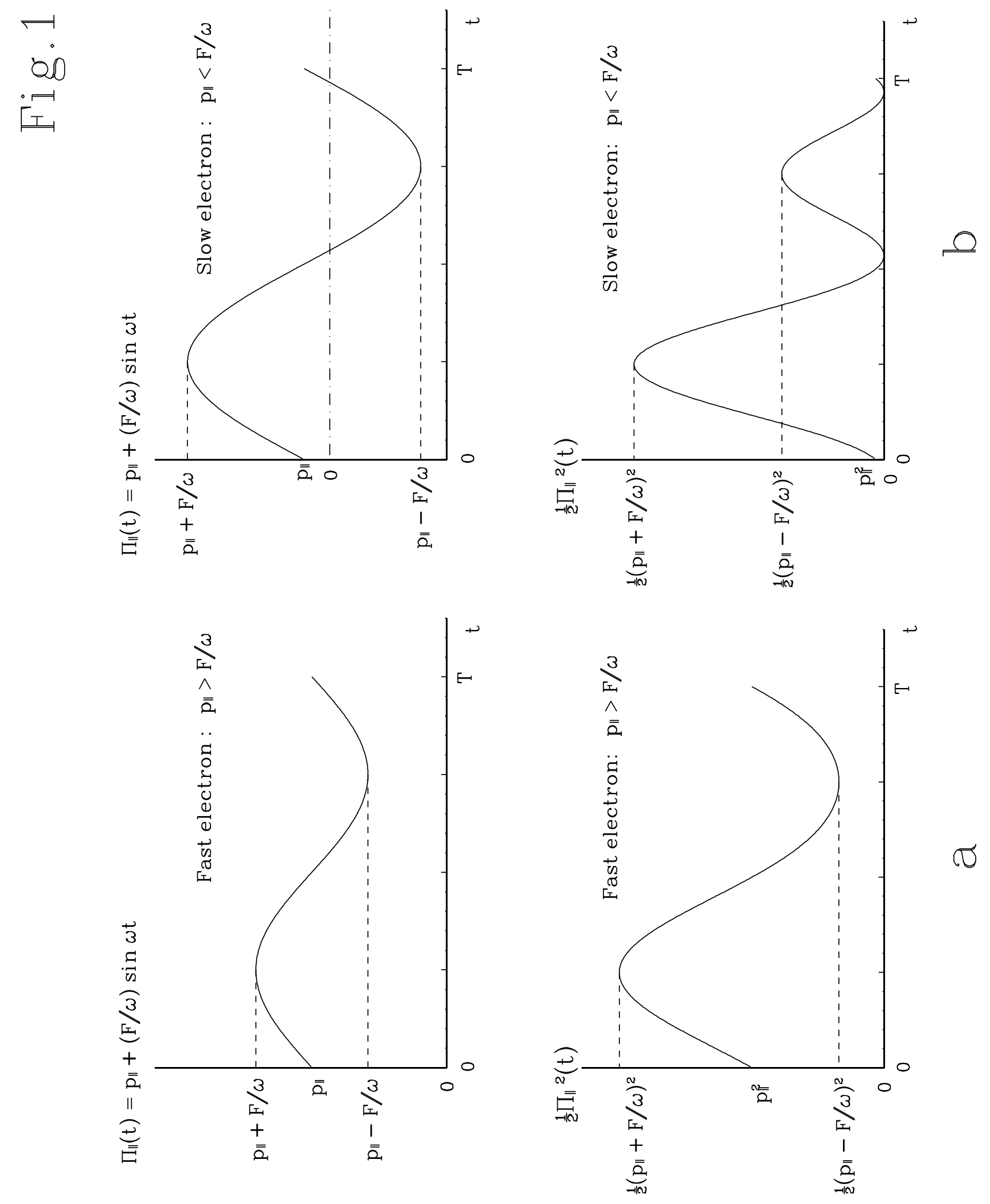


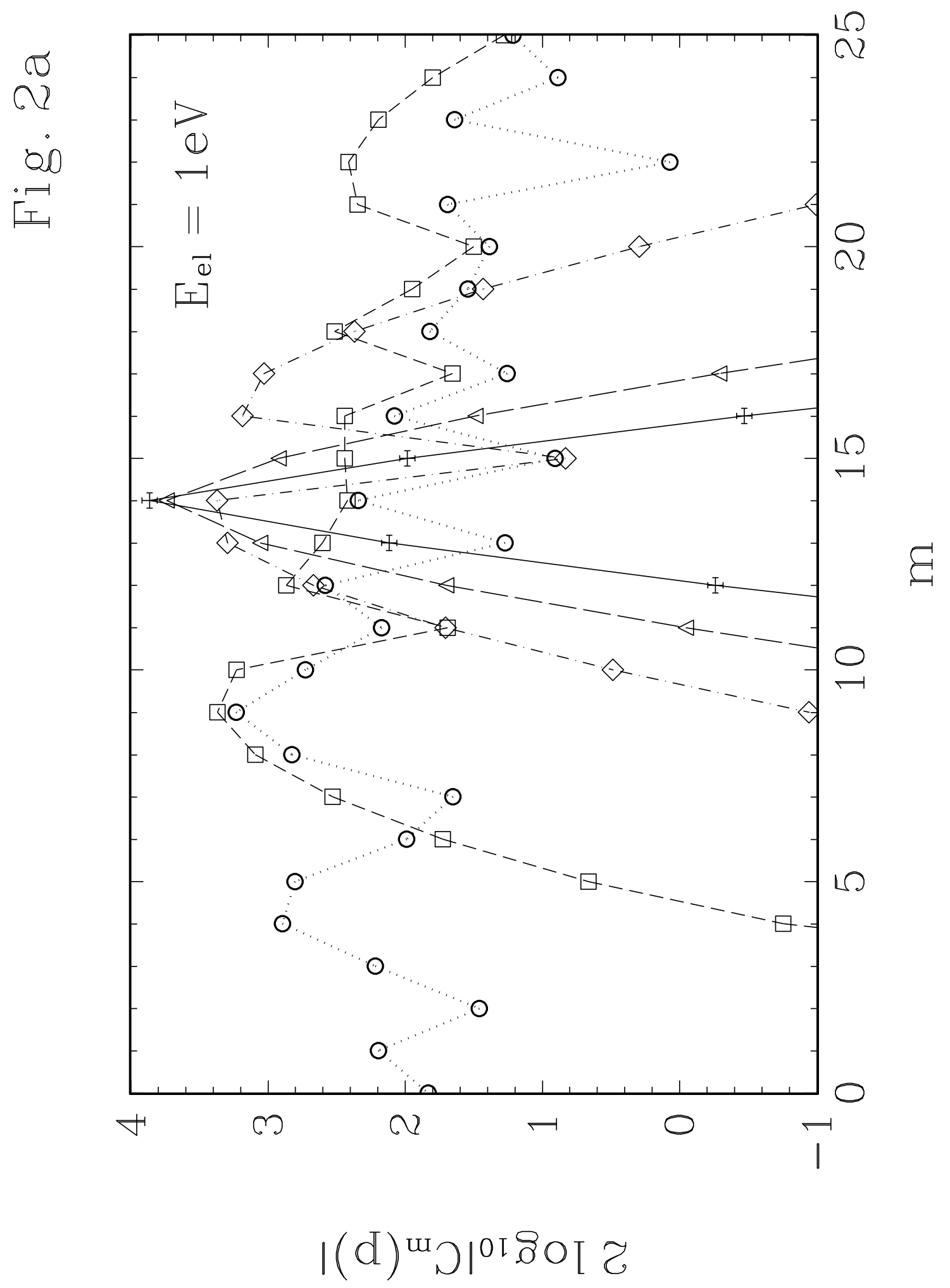




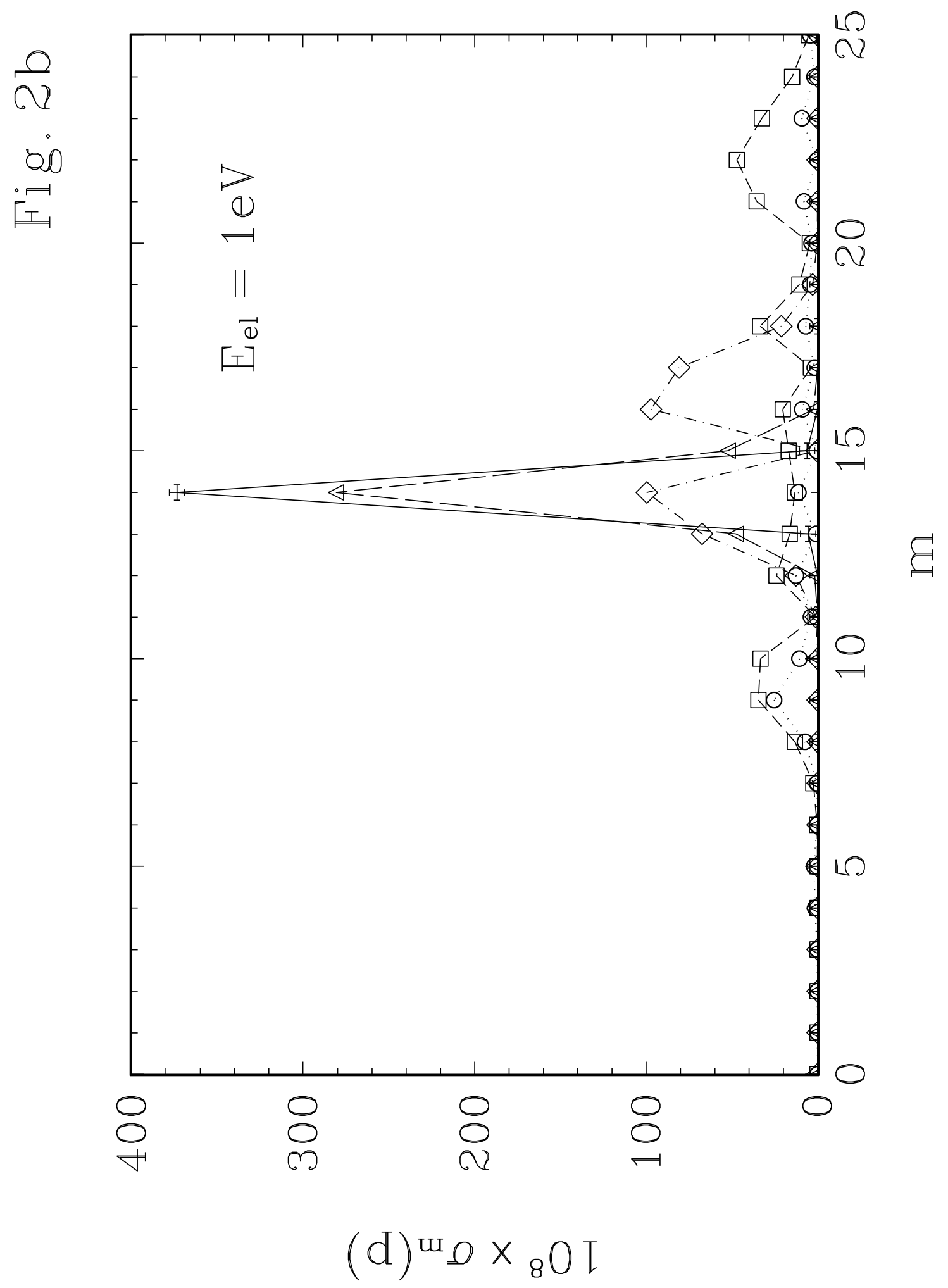




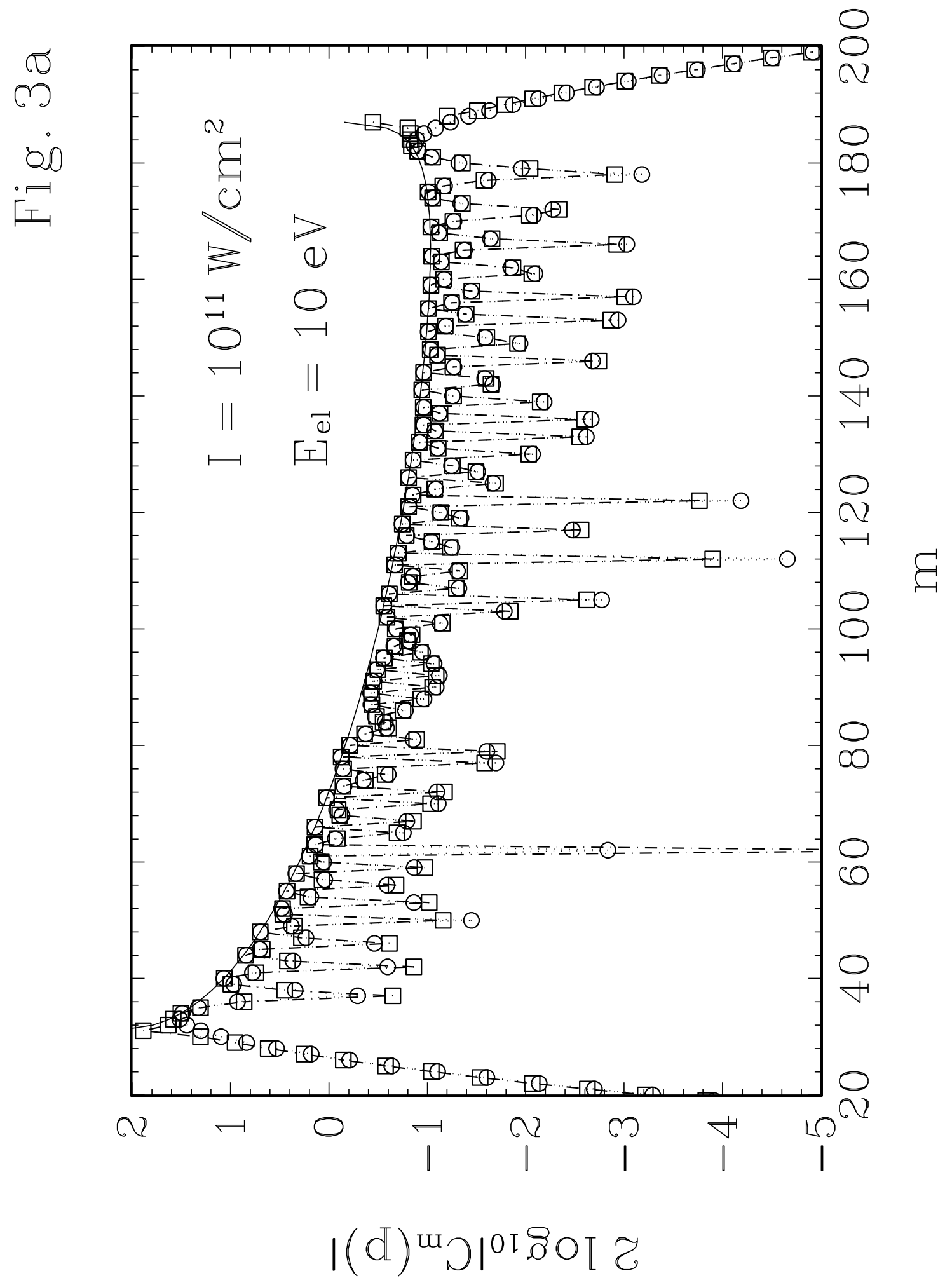




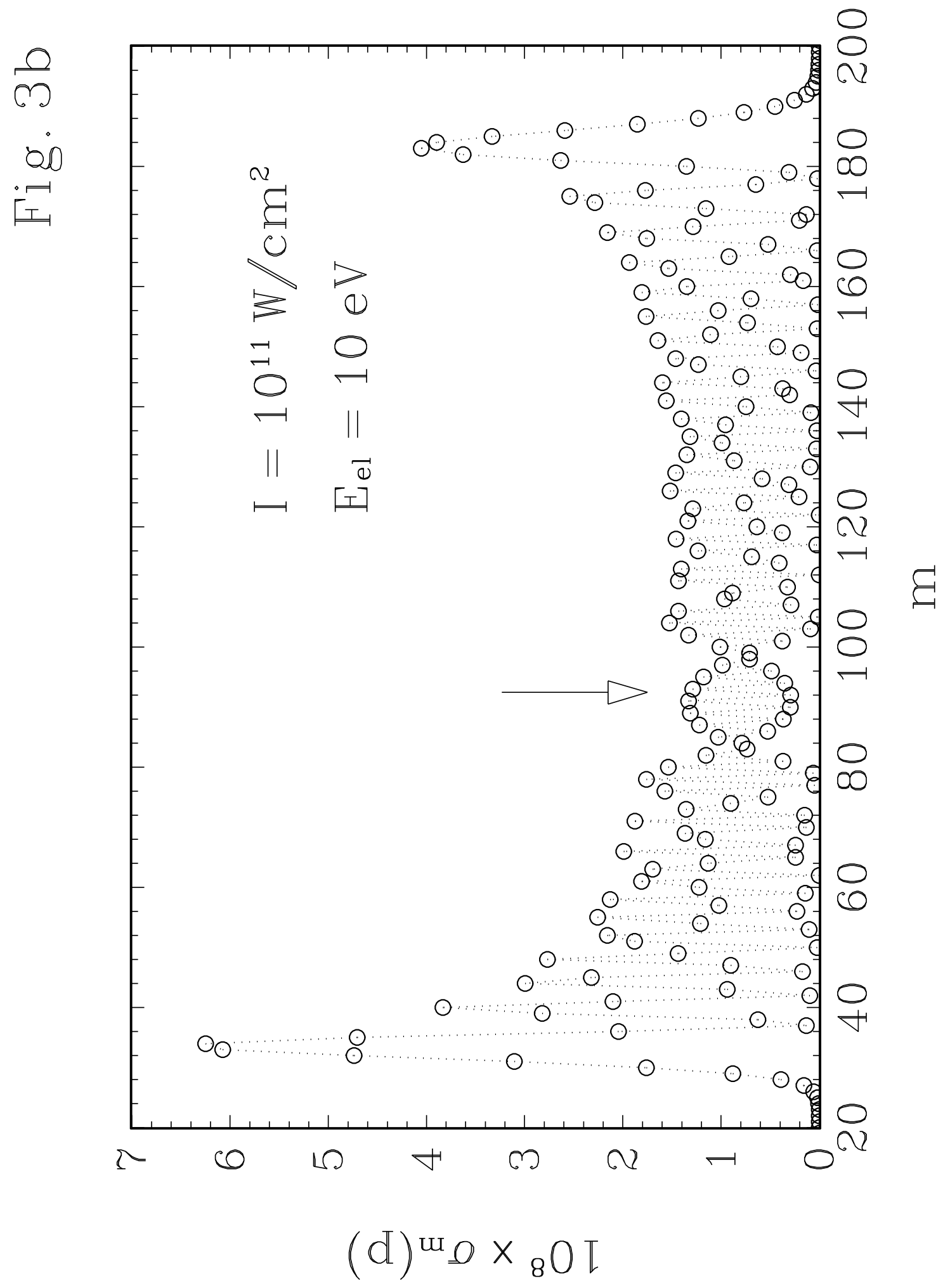




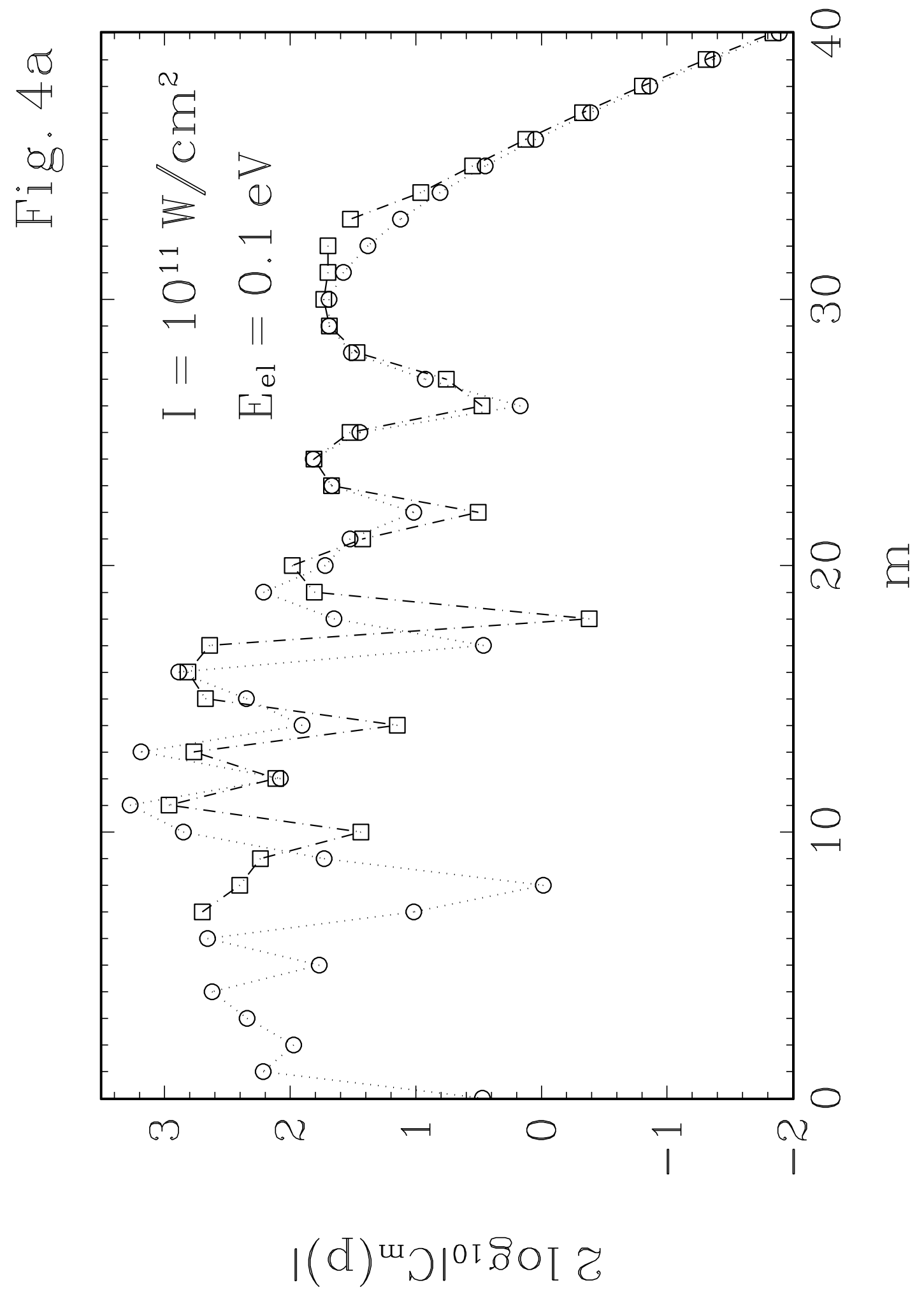




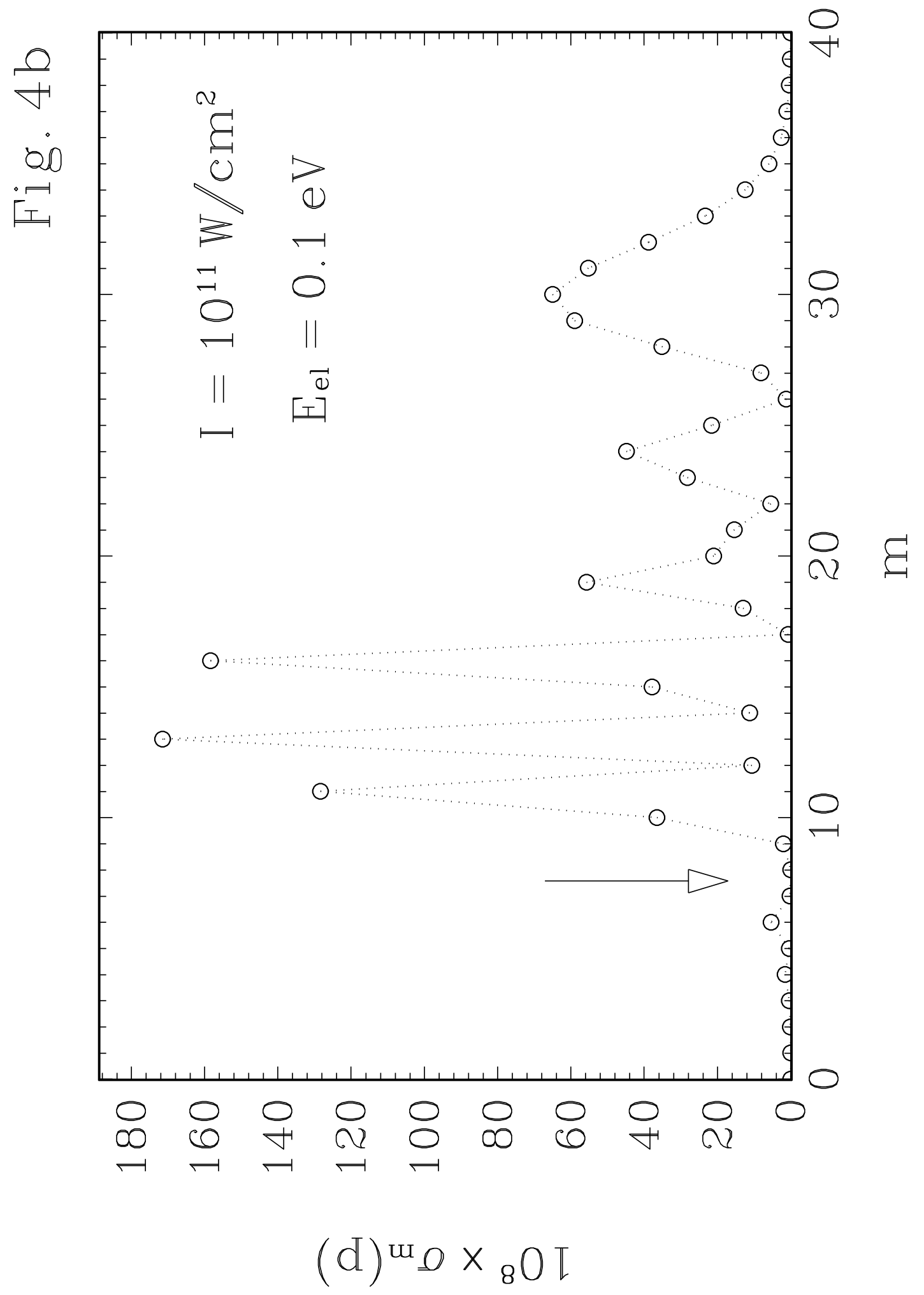




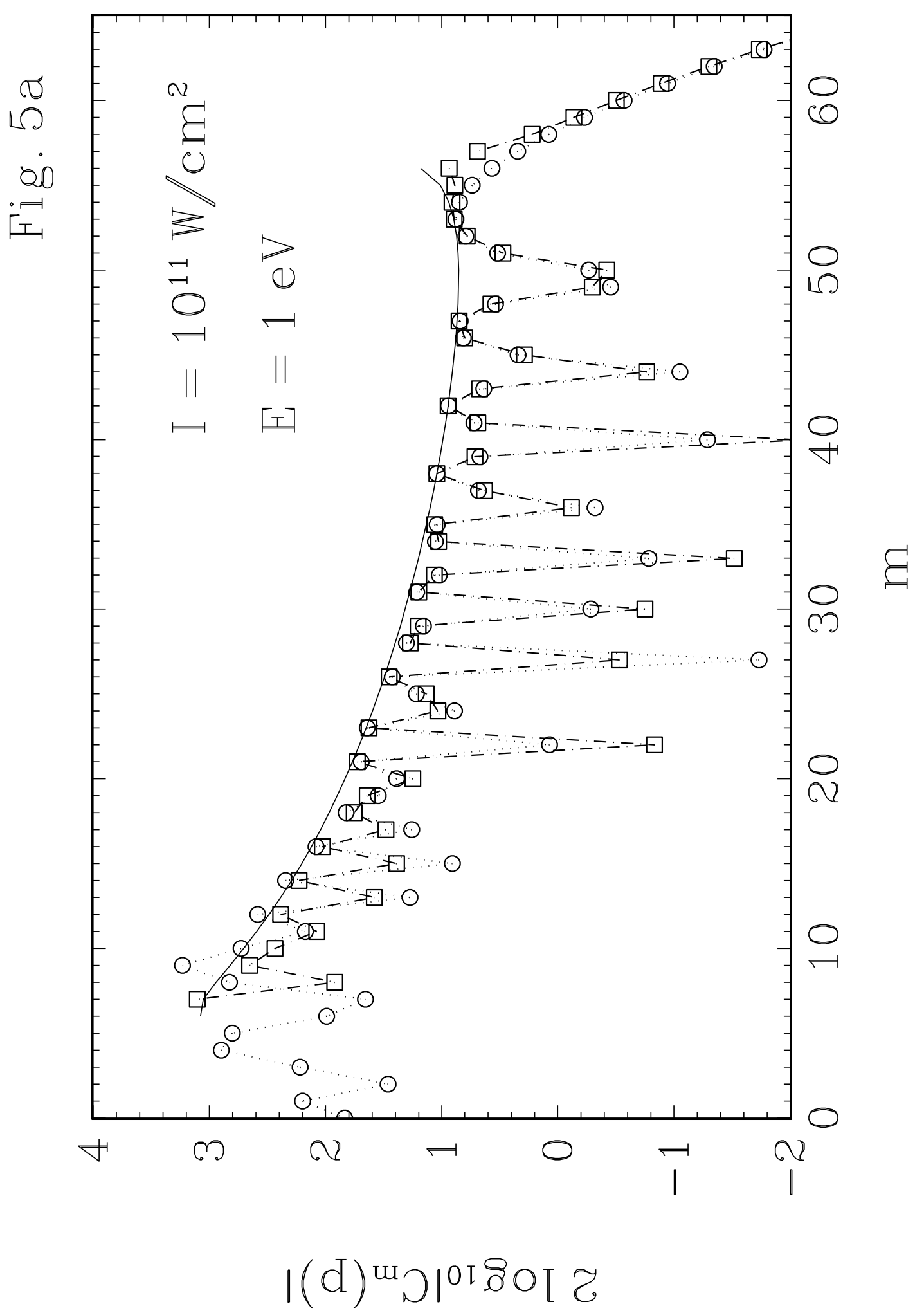




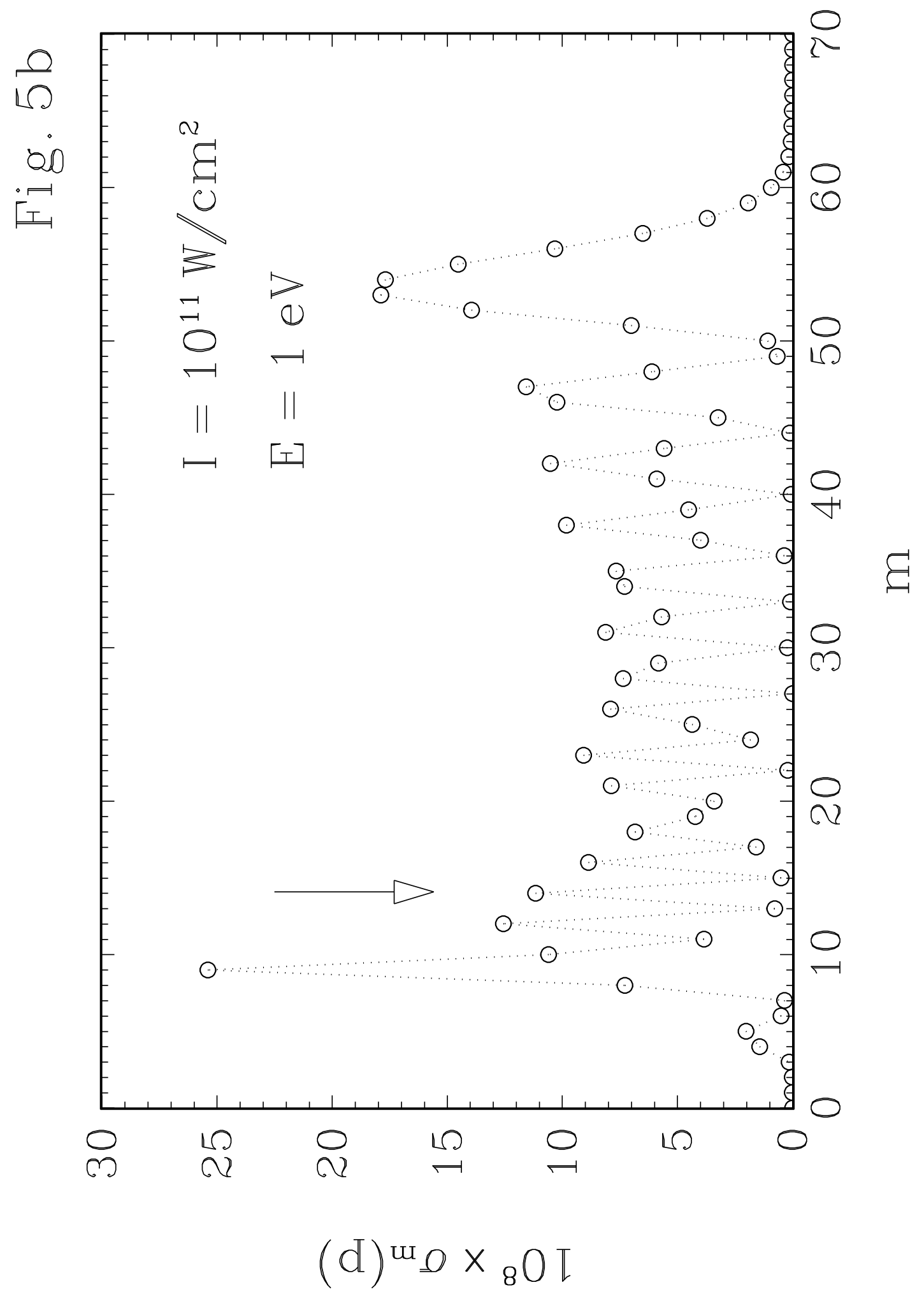

